\title{
Reflexiones en torno a la necesidad de cultivar competencias tecnológicas en docentes de educación superior desde una perspectiva pedagógica
}

\section{Reflections on the need to cultivate technological competence in higher education teachers from a pedagogical perspective}

\author{
Luz Marleny Cifuentes Osorio ${ }^{1}$ y Miguel Francisco Crespo Alvarado ${ }^{2}$ \\ ${ }^{1}$ Maestría en e-Learning, Universidad Autónoma de Bucaramanga, Bucaramanga, Colombia \\ ${ }^{2}$ Grupo Interdisciplinario de Investigaciones Sistémico Interpretativas S.C., Torreón, México \\ marlenycifuentes@gmail.com, migcrespo@hotmail.com
}

(Recibido: 11 mayo 2019; aceptado: 18 julio 2019)

\begin{abstract}
Resumen
El desarrollo de competencias en el uso pedagógico de las Tecnologías de la Información y las Comunicaciones (TIC) va más allá del simple cultivo de destrezas que permitan utilizar con relativa fluidez los dispositivos tecnológicos. Supone la posibilidad de reflexionar sobre su pertenencia en cada situación de clase, incluso cuando se trata de programas virtuales. Se trata de no olvidar que la educación no es un fin en sí mismo, sino un medio para alcanzar determinados propósitos y las TIC son, en ese sentido, también un medio del que puede o no echar mano quien educa. Esta investigación permitió diseñar y validar un Recurso Digital Interactivo (RDI) desde un escenario online, con el objetivo de fortalecer las competencias TIC desde la dimensión pedagógica en docentes de educación superior. El recurso conjuga los elementos del modelo instruccional ADDIE y las condiciones y principios de un DUA para su implementación y validación.
\end{abstract}

Palabras clave: Recurso Digital Interactivo (RDI), Modelo ADDIE, Diseño Universal de Aprendizaje (DUA).

\begin{abstract}
The development of competencies in the pedagogical use of Information and Communication Technologies (ICT) goes beyond the simple cultivation of skills that allow technological devices to be used with relative fluency. It involves the possibility of reflecting on your membership in each class situation, even when it comes to virtual programs. It is about not forgetting that education is not an end in itself, but a means to achieve certain purposes and ICTs are, in that sense, also a means that the educator may or may not help. This research allowed to design and validate an interactive digital resource (RDI) from an online scenario, with the aim of strengthening ICT skills from the pedagogical dimension in teachers of higher education. The resource combines the elements of the ADDIE instructional model and the conditions and principles of a DUA for its implementation and validation.
\end{abstract}

Keywords: Interactive Digital Resource (RDI), ADDIE Model, Universal Learning Design (ULD).

\section{Introducción}

Este artículo surge de la investigación propia de un trabajo de grado (Cifuentes Osorio, 2019) realizada en el seno de la Maestría en e-Learning de la Universidad Autónoma de Bucaramanga y la Universitat Oberta de Catalunya, cuyo producto central es el diseño de un Recurso Digital Interactivo (RDI) creado para fortalecer las competencias tecnológicas, desde la dimensión pedagógica, en docentes de educación superior. El propósito de este documento es dar cuenta de las circunstancias culturales que brindaron sentido

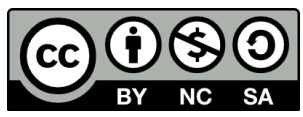


a la construcción del curso en cuestión y que trascienden la mera necesidad de desarrollar destrezas para el uso de dispositivos tecnológicos.

El método que se siguió para el diseño del RDI implicó, primero, un diagnóstico de las circunstancias actuales que atraviesa la incorporación de las TIC a la educación superior en el contexto de la cultura occidental del presente, con la idea de que el RDI a ser diseñado atendiera el desarrollo de competencias tecnológicas desde la dimensión pedagógica, sin descuidar el contexto que a estas les brinda sentido. Luego, apoyados en el modelo ADDIE se realizó el diseño. La razón fundamental para el empleo de dicho modelo es su carácter cíclico, que permite asumir lo diseñado como un producto no acabado que requiere ser ajustado de manera permanente. Finalmente, antes de ser presentado de manera formal se procedió a un pilotaje del RDI con resultados satisfactorios en términos de validación de su diseño.

Lo que aquí será expuesto es una serie de reflexiones en distintas capas que intentan mostrar una comprensión del problema de incorporar tecnologías a la educación, el cual conduce a la búsqueda de soluciones entre las que se incluye un cierto tipo de formación docente. El discurso no parte desde una postura que se oponga al uso de dispositivos tecnológicos; todo lo contrario, surge de la convicción de que estos deben convertirse en parte central del quehacer educativo, pero para ello es indispensable que se les cuestione de manera sistemática y permanente, lo que no necesariamente está sucediendo en la actualidad.

El predominio de una mirada ingenua y descuidada en torno a las tecnologías, y en particular a las tecnologías de la información y las comunicaciones (TIC), puede poner en peligro su potencial educativo, como también puede hacerlo un mal entendimiento de la dimensión pedagógica. Por eso, en este artículo también se discute y reflexiona en torno a ese concepto al que no se le suele poner atención cuando se trata de formación docente en tecnologías. Asimismo, se identifican las competencias relacionadas con dicha dimensión pedagógica de acuerdo con los estudios de la Unesco y de otras investigaciones en la materia, para fortalecer la idea del papel del recurso digital interactivo diseñado para alcanzar su propósito.

El artículo cierra con unas breves conclusiones tendientes a iluminar los esfuerzos por cultivar en los docentes competencias TIC desde la dimensión pedagógica.

\section{El problema de incorporar las TIC a la educación}

La cuestión en torno a la incorporación de la tecnología a los procesos educativos es, por lo regular, tratada como si ya se hubiera resuelto, como si todo mundo tuviera claridad en los porqués y los para qué, como si todas las dudas respecto a los qué, cuándo, cómo y dónde hubieran sido zanjadas y todo lo que hiciera falta fuera ponerse en acción. Sin embargo, estamos realmente lejos de tener respuestas contundentes a todas esas preguntas. La inquietud crece cuando atestiguamos el uso -o el "abuso"- que se da a las tecnologías en las aulas y otros espacios educativos, incluyendo los de la virtualidad.

Lejos de desaparecer, las didácticas expositivas se han generalizado con el desarrollo y la sofisticación de las TIC. Un estudio elaborado en México por Beatriz Zempoalteca y otros investigadores muestra cómo los medios tecnológicos más utilizados por docentes de educación superior en aquel país son los destinados a reforzar sus exposiciones (79\%) sólo por debajo de aquellos que les permiten realizar búsquedas en la Internet (86\%) (Zempoalteca Durán, Barragán López, González Martínez, y Guzmán Flores, 2017). Sus resultados coinciden con los encontrados en Costa Rica dos años antes por Abarca Amador (2015), quien encontró que el $100 \%$ de los docentes utilizaba las TIC que permiten ver videos, mientras que $92 \%$ empleaban aquellas destinadas a la presentación con diapositivas. En Colombia la realidad no debe ser muy distinta, aunque en el momento de elaborar el presente documento no contábamos con información precisa.

Que el uso de las TIC esté siendo aplicado de manera mayoritaria al refuerzo de las didácticas expositivas no sería tan problemático si quienes tienen a cargo las exposiciones contaran con las competencias necesarias para hacer de sus soliloquios algo impactante, apoyándose en las tecnologías para tal fin. Sin embargo, lo que solemos observar es lo contrario: monólogos planos y grises que se acompañan de diapositivas cargadas de textos, muchas veces plagiados o que ni siquiera se tuvo la precaución de leer con antelación, de tal manera que el docente va descubriendo su contenido al mismo tiempo que hace su lectura frente al grupo.

Es necesario recalcar, además, que la rutina de repartir temáticas para que los alumnos de manera individual o por equipos se hagan cargo de enseñarlos a sus compañeros no es sino una variación de esas formas expositivas, cuyas desventajas no se diluyen al corresponsabilizar a los estudiantes. Por el contrario, algunas clases en las que los alumnos son los que exponen pueden ser totalmente desmotivadoras para 
quienes las tienen que escuchar. ¿Se puede exigir, ante una experiencia como la descrita, que la atención se otorgue al expositor y no al teléfono móvil o a cualquier otro expositor?

En los escenarios virtuales hay también problemas. No son pocos los docentes que desconocen cuál debe ser su rol en los cursos en línea y que se repliegan en su papel para convertirse en simples revisores de tareas, desaprovechando las posibilidades que les ofrecen las herramientas tecnológicas, no solo por desconocimiento de estas, sino también porque, aunque sepan que existen, no tienen claridad de cuál es su potencial educativo o carecen de las competencias necesarias para optimizar su empleo.

La situación puede ser todavía peor cuando ni siquiera la tarea de evaluador de trabajos se desempeña de manera adecuada. No son escasas las experiencias de profesores que regresan a sus estudiantes los trabajos sin haber realizado una revisión y retroalimentación profunda del contenido, limitándose a revisar los aspectos formales como el tamaño de las márgenes, el tipo de fuente empleada o el número de referencias citadas, aspectos que sin duda son importantes, pero que no inciden en el desarrollo de conocimientos significativos para el alumnado, que desconoce si lo que aprendió es adecuado y pertinente. Ante esa lamentable realidad, no debe extrañar que los estudiantes de la educación virtual experimenten una profunda sensación de soledad y abandono, perdiendo así el entusiasmo inicial que los condujo a inscribirse en un curso.

Un estudio publicado en 2018 muestra un panorama poco alentador en Colombia sobre las prácticas que las Instituciones de Educación Superior (IES) ofrecen para mejorar sus competencias TIC: acceso a plataformas y repositorios; utilización de diferentes fuentes; difusión de experiencias docentes y la participación en foros, redes profesionales y grupos de innovación en general reciben una calificación promedio menor a 6,0 y se concentran, sobre todo, en aquellas destinadas solo al uso de la tecnología, dejando de lado su mayor comprensión y desarrollo (Melo Hernández, Gascó, Llopis, y González-Ramírez, 2018).

Sin embargo, desarrollar competencias para el uso pedagógico de las TIC va más allá del simple cultivo de destrezas que nos permitan utilizar con relativa fluidez los dispositivos tecnológicos. Supone, entre otras cosas, la posibilidad de reflexionar sobre su pertenencia ante cada situación de clase, incluso cuando se trata de programas virtuales, para potenciar las ventajas y disminuir los riesgos asociados a su empleo. Se trata de no olvidar que la educación no es un fin en sí mismo, sino un medio para alcanzar determinados propósitos y que las TIC son, en ese sentido, una herramienta de la que puede o no echar mano quien educa.

Pero, ¿por qué es tan problemático el uso irreflexivo de la tecnología en educación y qué hace tan necesario el desarrollo de competencias para su uso pedagógico? Esa pregunta no puede ser encarada de manera correcta si no se posee una conciencia de época, aunque sea incipiente. ¿Qué está ocurriendo en el mundo contemporáneo y qué papel juegan las tecnologías en ese devenir?

Como concuerdan diversos pensadores, Occidente se encuentra ante una crisis signada por el dominio de una racionalidad instrumental (Fuenmayor, 1994; Habermas, 1982) que dificulta la posibilidad de establecer fines trascendentes para la acción humana. La situación más común en la que se manifiesta ese obstáculo es la confusión de los fines con sus medios. Todo aquello, como la educación o las tecnologías, cuya naturaleza es la de ser medio, muy rápidamente comienza a ser abordado como si se tratase de un fin en sí mismo, creando la ilusión sobre su necesidad, cuando su empleo es siempre una entre varias opciones.

Esa muestra más reconocible del deterioro de la cultura occidental tiene, por lo menos, otras dos caras menos evidentes: la primera es la de la supresión de lo mediato para convertirse en inmediato; es decir, sin aquello que conecta el estado inicial con el final en lo-que-sea-el-caso. Un modelo general de educación supondría que quien se educa parte de un perfil inicial o de ingreso para alcanzar un perfil final o de egreso (ver Figura 1).

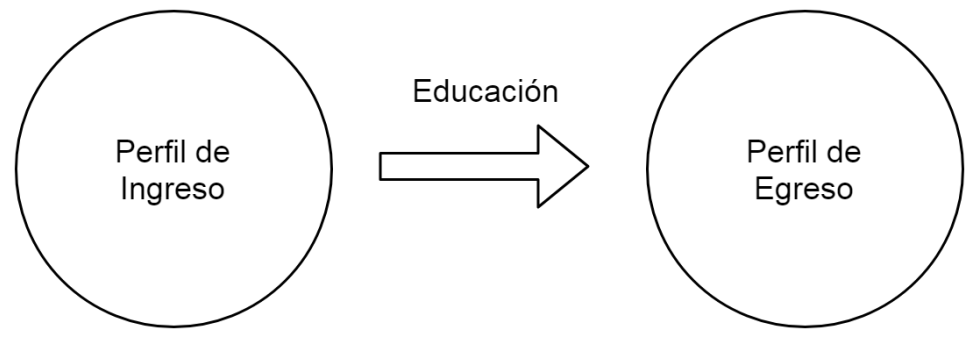

Figura 1. Modelo de educación 
Lo que está en medio, lo que conduce de un estado al otro es eso que llamamos educación. Sin embargo, con la crisis de la cultura occidental en marcha, la exigencia demandante es hacia la inmediatez: los estudiantes, los docentes y las instituciones empujan cada vez más la idea de que el mejor aprendizaje es el que ocurre en el menor tiempo posible. La confusión radica en que, al no distinguirse el medio del fin, el medio logra colarse como propósito porque es aquello con lo que se tiene el contacto más próximo: la herramienta siempre está a la mano, lo que se podría lograr con esta no (el hecho de que exista en potencia no significa que esté realizado).

La segunda cara del deterioro cultural es todavía menos evidente que la anterior: consiste en un empobrecimiento del Ser que conduce a lo-que-sea-el-caso a manifestarse en exclusiva como un dispositivo listo para su empleo (Fuenmayor, 2016; Heidegger, 1977). Para decirlo de forma sencilla, para nosotros: hombres y mujeres de la cultura occidental en el presente, todo lo existente -y no solo los aparatos tecnológicos- se nos ofrece como algo que está allí dispuesto para ser utilizado a nuestro arbitrio. Las computadoras, sus programas, pero también las flores, las montañas, las personas, sus ideas, emociones y creencias, todo, absolutamente todo lo existente, luce primordialmente como un recurso. He aquí una razón más poderosa para develar por qué los fines se desdibujan.

Hay distintas versiones que dan cuenta de cómo fue que lo existente llegó a ser simplemente un dispositivo listo para ser empleado (Crespo Alvarado, 2013; Roldán, 2005; Villareal, 2007). Lo importante aquí es dejar sentado qué se entiende cuando se reclama una cierta conciencia de época como requisito indispensable para responder a la pregunta por lo problemático que resulta el uso irreflexivo de las tecnologías en educación y por la necesidad de cultivar competencias en los docentes, desde la dimensión pedagógica, para su empleo.

Según Crespo (2015), la forma dominante como se están empleando las TIC en educación fomentan y profundizan las expresiones del deterioro cultural de Occidente que hemos descrito; de ahí que, sin renunciar a su empleo -lo que carecería de todo sentido-, sí es indispensable hacer un uso consciente de los peligros a los que exponemos a la cultura en la que estamos incidiendo con nuestro esfuerzo educativo.

\section{La dimensión pedagógica del uso de las TIC en educación}

Como sucede con cualquier fenómeno, la aproximación a las TIC puede hacerse desde distintos enfoques o dimensiones. Quienes las desarrollan, por ejemplo, suelen observarlas desde una perspectiva ingenieril que comprende apenas las generalidades sobre sus posibles usos. Pero incluso los docentes están ante la posibilidad de abordar el conocimiento de las tecnologías y los dispositivos desde ángulos que no son pedagógicos, como sucede con aquellos que las emplean para sus controles escolares o para la elaboración de los materiales que utilizarán en clase.

Como señala la Unesco, una dimensión pedagógica de las TIC en educación consiste en toda labor docente relacionada con la capacidad para apoyar el aprendizaje significativo y el desarrollo integral de los estudiantes a través de la creación de prácticas, actividades llenas de sentido para los que participan en ellas, el reconocimiento de problemáticas disciplinares o del entorno, la generación de experiencias que promuevan relaciones concretas con las problemáticas identificadas, la promoción de la reflexión y del pensamiento crítico y la evaluación integral del aprendizaje. De la misma manera, implica la vocación para la formación de personas, el manejo innovador y creativo de recursos tecnológicos y metodologías para la enseñanza y la evaluación, así como la habilidad para generar impacto e influencia, escuchar, preguntar, explicar y comunicar de manera efectiva (Organización de las Naciones Unidas para la Educación la Ciencia y la Cultura - Unesco, 2016).

Detengámonos brevemente en esa definición. Observemos, primero, que hay un propósito establecido con claridad: el aprendizaje significativo y el desarrollo integral de los estudiantes. Este, por supuesto, es a su vez un medio, pues las sociedades no educan a sus individuos por mero hábito, sino que están esperando una mejoría colectiva gracias a ese esfuerzo, sin importar los términos en que esta sea entendida por cada grupo humano diferente. Ese "desarrollo integral" se logra, de acuerdo con la definición, mediante una amplia gama de competencias que hay que cultivar en los estudiantes, para lo cual las tecnologías se ofrecen como un apoyo.

¿Qué significa, por ejemplo, crear una práctica? ¿Qué implica el diseño de actividades "llenas de sentido" para quienes practican en ellas? ¿Cómo lograr el "reconocimiento de problemáticas" en los estudiantes? ¿Cómo se generan “experiencias que promuevan relaciones concretas” con esas problemáticas? ¿Es posible promover la reflexión y el pensamiento crítico? ¿Qué caminos se deben seguir para la evaluación 
integral de los aprendizajes? ¿Cuál es el papel que, en todo lo anterior, pueden jugar las TIC? Las anteriores son algunas preguntas que solo podrían ser lanzadas desde la dimensión pedagógica y que son necesarias para fomentar el uso reflexivo de las TIC en educación.

Estamos, entonces, ante un argumento más que apoya la necesidad de preparar al docente para el uso de las tecnologías; pero, además, ante uno que demuestra que el conocimiento técnico no basta. Como fácilmente puede interpretarse de la propuesta de la Unesco, es necesario que un interés crítico emancipatorio (Habermas, 1982) oriente los esfuerzos de aproximación a las TIC como fenómeno, así como de sus posibles usos educativos. Solo de esa manera se podría garantizar que los propósitos establecidos para la dimensión pedagógica son, en verdad, perseguidos. Para que su búsqueda -necesariamente incompletapueda ser satisfactoria se requiere del cultivo de las competencias adecuadas. ¿Cuáles son?

\section{Competencias de la dimensión pedagógica}

Desde que el uso del concepto competencia comenzó a extenderse, sobre todo en educación superior, fue aceptada la distinción entre las competencias científicas, propias de la disciplina de formación de quienes profesan en las Instituciones de Educación Superior (IES), y las asociadas al ejercicio de la docencia (Bozu y Canto Herrera, 2009; Valcárcel, 2003). Con ello se reconoce que una cuestión es dominar un campo del conocimiento humano y otro asunto diferente es estar preparado para cultivar ese saber en otros. Ahora, se hace necesaria una nueva distinción, estrechamente relacionada con las competencias de la docencia, que refiere a las capacidades necesarias para hacer un uso reflexivo de las TIC en educación. Como señalan Villalobos Hernández, Torres y Barona (2014) en el caso específico de la educación virtual, "el proceso de formación del tutor virtual es un tema educativo relevante que, actualmente, se encuentra en construcción" (p.154). De ahí la necesidad de reflexionar e identificar las competencias necesarias para su correcto desempeño.

La Unesco (2016) identifica tres competencias propias de la dimensión pedagógica, que consisten en la capacidad del docente para diseñar escenarios educativos apoyados en TIC que posibiliten el logro de aprendizajes significativos y el desarrollo integral, implementar experiencias educativas y evaluar la efectividad de sus acciones a partir de lo aprendido de manera significativa por los estudiantes (Organización de las Naciones Unidas para la Educación la Ciencia y la Cultura - Unesco, 2016). Esas competencias están fundamentadas en tres niveles de apropiación de las TIC (integración, reorientación y evolución), que a su vez se rigen por la forma como cada docente conoce, utiliza y transforma los procesos de formación que conducen a mejorar las prácticas educativas basadas en las TIC. Para esto último, el organismo internacional definió una serie de estándares que ofrecen algunos descriptores para identificar esas buenas prácticas y distinguirlas de posibles usos educativos poco deseables.

Existen, por supuesto, otros esfuerzos que vale la pena considerar en el intento por definir qué competencias necesita adquirir un docente, desde la dimensión pedagógica, para que sea exitosa la incorporación de las TIC en los procesos educativos. El trabajo de Ardila Rodríguez (2009) es importante en ese sentido porque logra revelar que, desde la perspectiva de los docentes, el tipo de competencias menos importante para desempeñarse de manera adecuada en entornos virtuales es el relacionado con el diseño gráfico en la presentación de contenidos. En contraste, las competencias de mayor relevancia son aquellas que permiten la incorporación de las TIC a "los procesos de comunicación, asesoría, seguimiento y acompañamiento a estudiantes” (Ardila Rodríguez, 2009, p.10).

Otro esfuerzo destacado es el realizado por Urdaneta (2008), quien determinó un perfil de competencias del docente-tutor en línea de educación universitaria, luego de constatar que, en general, las IES se han preocupado por favorecer las condiciones de infraestructura en tecnologías en diferentes instancias, incluyendo la académica, pero han dejado de lado la formación de los docentes en el desarrollo de procesos de aprendizaje bajo las mediaciones tecnopedagógicas que ofrece el uso de las tecnologías en la educación.

Son cuatro las competencias que determinaron como necesarias: a) pedagógica, entendida como la capacidad para explicar y escenificar procesos de enseñanza-aprendizaje requeridos en educación virtual; b) comunicativa, que es la habilidad para intervenir e interponer acciones didácticas que conduzcan al desarrollo de competencias en otros; c) psicológica, la capacidad para comprender los procesos internos de los otros y aprovecharlos para facilitar su aprendizaje, y d) técnica, que permite al docente desempeñarse de manera exitosa en un entorno tecnológico.

Carmen Yot, Carlos Marcelo y Víctor Hugo Perera (2016), de la Universidad de Sevilla, realizaron un estudio sobre las tareas y competencias del tutor online. Los resultados muestran que son competencias 
clave: a) aquellas que permiten recibir al alumno nuevo para familiarizarse con él y, a su vez, que este se familiarice con el entorno mediado tecnológicamente y con las competencias que serán desarrolladas; b) las que posibilitan crear un ambiente de aprendizaje y comunidades estudiantiles que puedan trabajar de manera colaborativa en dicho escenario; c) las que le permiten supervisar que los materiales diseñados para el estudiante sean empleados de manera adecuada y que las actividades conduzcan a aprendizajes sólidos; y d) las que hacen posible un seguimiento puntual de los estudiantes, de su desempeño y de los logros que van adquiriendo a través del proceso.

A partir de un estudio realizado por Hernández Suárez, Gamboa Suárez, y Ayala García (2014) se determinó que, además de las competencias comúnmente asociadas al manejo de las TIC, los docentes deben desarrollar competencias genéricas e instrumentales, metodológicas, tecnológicas, investigativas, lingüísticas, sistemáticas y de liderazgo.

Estos son solo unos pocos ejemplos de los intentos por determinar un listado de competencias que deben desarrollar los docentes para que la dimensión pedagógica en la incorporación de las tecnologías a la educación pueda ser atendida de manera exitosa. Los casos aquí presentados permiten iluminar el amplio espectro que existe y que encuentra su punto común en la conciencia, cada vez mayor, de que se necesitan más y mejores habilidades para lidiar con ese desafío. Hagamos un pequeño paréntesis en nuestro discurso para reflexionar sobre lo expuesto hasta ahora.

\section{Tres reflexiones en torno a la búsqueda de competencias desde la dimensión pedagógica del uso educativo de las TIC}

Existen tres asuntos clave para destacar en torno a la manera como hasta ahora se han planteado las competencias para la dimensión pedagógica de las TIC en educación.

El primero tiene que ver con la proclividad a pensar-actuar el tema desde una perspectiva reduccionista, como si educación y tecnologías no fueran fenómenos culturales amplios que permean la totalidad de la existencia de los seres humanos contemporáneos. Es muy comprensible que la necesidad de analizar nos conduzca a enfocarnos en los elementos clave de un determinado proceso, sobre todo cuando la tradición del pensamiento occidental nos conduce a aislar el problema para poder atenderlo (Descartes, 2009). Sin embargo, tal ejercicio debería mostrar con gran fuerza que se reconocen las limitaciones de abordar así los temas de investigación.

La reducción del fenómeno de la incorporación de las TIC a la educación olvida, por ejemplo, que la educación en sí misma libra sus propias batallas en medio de circunstancias que le son adversas, como la transformación ontológica de la relación maestro-aprendiz en una relación de tipo cliente-proveedor (Cabrera Cruz y Crespo Alvarado, 2005), la disputa entre las concepciones unificante y diversificante de la humanidad (Fuenmayor, 2000) y el fin de la época metafísica (Heidegger, 2011). Asimismo, deja de lado la discusión actual sobre la tecnología y la manera en que su presencia está alterando las experiencias de vida. Desde la óptica de nuestra investigación es indispensable mantener ese escenario en mente cada vez que se discuten los asuntos concernientes a la incorporación de las TIC a la educación.

La segunda reflexión, vinculada a la forma como intentan determinarse competencias para la dimensión pedagógica del uso de las TIC en educación, tiene que ver con el conflicto actual entre el afán de aseguramiento y la aceptación de que cada quien comprende a su modo (de ahí el uso frecuente que, en el discurso de las competencias, se da a la idea "apropiación"). En ese sentido, las propuestas se presentan bajo la pretensión de garantizar determinados resultados; sin embargo, lo que se consigue son sólo aproximaciones ante la imposibilidad de predecir el futuro, sobre todo cuando se trata de los seres humanos (MacIntyre, 1987). No es que los planteamientos sean del todo inconscientes con respecto a la diversidad de posibilidades que obtendrán por resultado si se realizan las acciones que sugieren, pero la aspiración de fondo continúa siendo, en muchos casos, la de controlar lo que sucederá.

El aparente conflicto se resuelve utilizando la idea de "apertura", que tiene sentido cuando se resuelve, por un lado, que si bien se está dispuesto a admitir una diversidad de resultados como aceptables, eso no significa que se puede estar de acuerdo con cualquier posible respuesta. Es decir, hay resultados que pasan y otros que no. La noción de apertura indica que hay una especie de puerta que deja entrar aquello que se apegue a ciertos mínimos e impide el paso de lo que, de plano, no encaje. Es verdad que las nociones de "indicador" y "descriptor", presentes de manera cotidiana en el discurso de las competencias, parecen apuntar a esa apertura; no obstante, necesitan mayor profundización y, más aún, requieren ser 
conscientemente utilizados en ese sentido, para que quede claro qué es lo que se desea asegurar: que los resultados se parezcan a los esperados.

El tercer asunto para reflexionar está asociado al fomento de la creatividad y la innovación. Como se ha dicho, en nuestro contexto social es de suma importancia romper con la dependencia tecnológica hacia otras naciones (Crespo Alvarado, 2015). Por tal motivo, y en concordancia con la idea de apertura expuesta anteriormente, es indispensable vigilar que las competencias cultivadas en docentes y estudiantes sean reformuladas de manera continua con la intención de acabar con ese hábito cultural de ser simples usuarios de las soluciones creadas en otras latitudes, las cuales suelen alejarse, en mayor o menor medida, de los problemas concretos que plantean nuestras realidades. La innovación y la creatividad, en ese sentido, suponen no limitarse a encontrar nuevos usos educativos a herramientas ya existentes, sino más bien potenciar la capacidad para desarrollar nuevos dispositivos y aplicaciones, bajo la convicción de que tal esfuerzo presume grandes avances en la adquisición de conocimientos y habilidades en quienes participan de él.

Con todo lo anterior en mente, ¿qué se debe hacer si se desea cultivar en los docentes las competencias necesarias para incorporar las TIC en la educación desde la dimensión pedagógica?

\section{Hacia el diseño de un recurso digital interactivo que fortalezca las competencias TIC}

Hasta ahora es posible concluir, por lo menos, dos asuntos puntuales: la necesidad de cultivar en los docentes de todos los niveles las competencias TIC desde la dimensión pedagógica y la enorme dificultad que dicha tarea representa y que no debería desdeñarse ni ser abordada con descuido. A quienes se disponen a realizar esfuerzos educativos de cualquier índole con el propósito de fortalecer el dominio de los docentes frente a las tecnologías y sus dispositivos, deben saber que el simple "aprender a hacer" no alcanza, dadas las circunstancias de nuestra cultura en el presente.

Además, resulta muy importante entender que los cursos mediados por tecnología deben ser, en sí mismos, ejemplos de un empleo adecuado de las TIC. Eso eleva la exigencia para los formadores de competencias tecnológicas en los docentes, quienes se verán forzados a dedicar tiempo de calidad a sus diseños instruccionales y a valerse de las mejores prácticas para su desarrollo. Por supuesto, también a mantener constante la reflexión en torno a la incorporación de las tecnologías a la educación y las oportunidades y peligros que ello representa.

Esa es la razón por la que se empleó el modelo ADDIE (Análisis, Diseño, Desarrollo, Implementación y Evaluación) para el curso diseñado en el proyecto de investigación del que forma parte este artículo, pues permite crear y organizar material educativo siguiendo pasos sistemáticamente organizados que, en todo momento, pueden conducir a una valoración sobre lo que se está realizando, y por ende a la mejora continua, dado su carácter cíclico.

En la educación virtual es imprescindible hacer énfasis en los principios de la instrucción para la elaboración y el diseño de materiales didácticos, que desarrollen estrategias y actividades para facilitar al docente y al estudiante el logro de las metas fijadas en la evaluación. El modelo ADDIE permite apoyar el proceso de la enseñanza por medio de la tecnología. De manera adicional, puede convertirse en un referente para los programas de educación a distancia y virtual, al ser un modelo versátil usado por los diseñadores instruccionales y también por las entidades corporativas, debido a la modalidad de la evaluación en cada fase.

De igual forma, el modelo ADDIE es una excelente herramienta de gestión para la creación de recursos interactivos digitales, cuidadosamente estructurados para el desarrollo de procesos de aprendizaje en una población amplia, debido a la claridad y a la organización con que se implementa cada fase en el ambiente virtual de aprendizaje o de forma presencial. Este modelo se puede desarrollar bajo la teoría del constructivismo, por la forma de integrar los procesos de aprendizaje del estudiante en cada fase del diseño instruccional.

Como resultado del ejercicio reflexivo aquí expuesto, se diseñó un RDI en el que, además de buscar fortalecer la dimensión pedagógica de las competencias necesarias para el uso de las TIC en educación superior, cuida, por un lado, evitar los peligros enunciados en este artículo, pero, además, fomentando la propia reflexión de los docentes, la cual es indispensable para un uso adecuado de las TIC en educación.

\section{Declaración de conflicto de intereses}

Los autores declaran no tener conflicto de intereses con respecto a la investigación, autoría y/o publicación de este artículo. 


\section{Referencias}

Abarca Amador, Y. (2015). El uso de las TIC en la educación universitaria: motivación que incide en su uso y frecuencia. Revista de Lenguas Modernas, (22). https://doi.org/10.15517/rlm.v0i22.19692

Ardila Rodríguez, M. (2009). Docencia en ambientes virtuales: nuevos roles y funciones. Revista Virtual Universidad Católica Del Norte, 28, 1-15. Recuperado de: http://revistavirtual.ucn.edu.co/index.php/RevistaUCN/ article/view/82/169

Bozu, Z., y Canto Herrera, P. J. (2009). El profesorado universitario en la sociedad del conocimiento: competencias profesionales docentes. Revista de Formación e Innovación Educativa Universitaria, 2(2), 87-97. Recuperado de: http://refiedu.webs.uvigo.es/Refiedu/Vol2_2/REFIEDU_2_2_4.pdf

Cabrera Cruz, J. D., y Crespo Alvarado, M. F. (2005). Il senso delle pratiche e la relazione maestro-apprendista oggi. In La Svolta Pratica In Filosofia: Dalla Filosofia Pratica Alla Pratica Filosofica (pp. 173-192). Quodlibet edizioni.

Cifuentes Osorio, L. M. (2019). Diseño de un curso-recurso digital interactivo para fortalecer las competencias TIC desde la dimensión pedagógica en docentes de educación superior en modalidad e-Learning. Universidad Autónoma de Bucaramanga.

Crespo Alvarado, M. F. (2013). Primeros trazos de una Historia-Ontológica de la Educación en América Latina, en la búsqueda de un Sistema Educativo para el presente que aspire al enriquecimiento del sentido. Universidad de los Andes, Mérida, Venezuela.

Crespo Alvarado, M. F. (2015). Formación de Prosumidores de Multimedia Educativa como Respuesta a la Imposibilidad del Sentido Holístico en el presente en la cultura occidental. Revista Colombiana de Computación, 16(2), 7-24. Recuperado de: https://revistas.unab.edu.co/index.php/rcc/article/ view/2549/2184

Descartes, R. (2009). Discurso del método (Edición Bilingüe). Colihue Clásica.

Fuenmayor, R. (1994). El olvido del sentido holístico en la época postmoderna. Revista Sistemas, (1), 15-30. Recuperado de: http://www.saber.ula.ve/bitstream/handle/123456789/15895/fuenmayor-olvido. pdf?sequence $=1$ yisAllowed $=y$

Fuenmayor, R. (2000). Sentido y sinsentido del desarrollo. Universidad de los Andes.

Fuenmayor, R. (2016). El cultivo de la verdad. Ibagué, Colombia: Ediciones Unibagué.

Habermas, J. (1982). Conocimiento e interés. Madrid: Taurus.

Heidegger, M. (1977). The Question Concerning Technology and Other Essays. Garland Publishing, INC.

Heidegger, M. (2011). Aportes a la filosofía. Acerca del evento. Buenos Aires: Biblos.

Hernández Suárez, C. A., Gamboa Suárez, A. A., y Ayala García, E. T. (2014). Competencias TIC para los docentes de educación superior. In Congreso Iberoamericano de de Ciencia, Tecnología, Innovación y Educación.

MacIntyre, A. (1987). Tras la virtud. Barcelona: Crítica.

Marcelo, C., Yot, C., y Perera, V. H. (2016). El conocimiento tecnológico y tecnopedagógico en la enseñanza de las ciencias en la universidad. Un estudio descriptivo. Enseñanza de Las Ciencias. Revista de Investigación y Experiencias Didácticas, 34(2), 67. https://doi.org/10.5565/rev/ensciencias.1552

Melo Hernández, M. E., Gascó, J. L., Llopis, J., y González-Ramírez, R. (2018). Prácticas de los docentes para ampliar las competencias en el uso de las Tecnologías de Información y Comunicación (TIC) en la educación superior en Colombia. In Octaedro (Ed.), El compromiso académico y social a través de la investigación e innovación educativas en la Enseñanza Superior (pp. 294-304). Recuperado de: http://hdl. handle.net/10045/87491

Organización de las Naciones Unidas para la Educación la Ciencia y la Cultura - Unesco. (2016). Competencias y estándares TIC desde la dimensión pedagógica: Una perspectiva desde los niveles de apropiación de las TIC en la práctica educativa docente. Recuperado de: http://www.unesco.org/new/fileadmin/ MULTIMEDIA/FIELD/Santiago/pdf/Competencias-estandares-TIC.pdf

Roldán, S. (2005). Esbozo de una historia-ontológica de la educación moderna y muestra del diseño de actividades pedagógicas para el 7o y 80 de educación básica (un aporte al Proyecto de Educación de la Sistemología Interpretativa). Universidad de los Andes, Mérida, Venezuela. 
Urdaneta, M. (2008). Perfil de competencias del docente como tutor en línea para la educación a distancia. Educación Superior, 9-34. Recuperado de: http://servicio.bc.uc.edu.ve/educacion/eduweb/vol2n2/art6.pdf

Valcárcel, M. (2003). La preparación del profesorado universitario para la convergencia europea en educación superior. Madrid.

Villalobos Hernández, M. M., Torres Velandia, S. Á., y Barona Ríos, C. (2014). Competencias docentes y formación de docentes virtuales. Revista de Transformación Educativa, 1, 136-157. Recuperado de: https://rete.mx/ attachments/article/8/RETE - Competencias docentes.pdf

Villareal, M. (2007). Inicio en la Grecia Clásica de una Historia Ontológica de la Educación Occidental y Muestra del Diseño de Actividades Pedagógicas para 1o, 2o, 3o Y 5o de Educación Primaria (un aporte al Proyecto de Educación de la Sistemología Interpretativa). Universidad de los Andes, Mérida, Venezuela.

Zempoalteca Durán, B., Barragán López, J. F., González Martínez, J., y Guzmán Flores, T. (2017). Formación en TIC y competencia digital en la docencia en instituciones públicas de educación superior. Apertura, 9(1), 80-96. Recuperado de http://www.udgvirtual.udg.mx/apertura/index.php/apertura/article/view/922/644 